\title{
Dependence Properties of B-Spline Copulas
}

\author{
Dependence properties of B-spline copulas
}

\author{
Xiaoling Dou • Satoshi Kuriki • \\ Gwo Dong Lin · Donald Richards
}

Received: date / Accepted: date

\begin{abstract}
We construct by using B-spline functions a class of copulas that includes the Bernstein copulas arising in Baker's distributions. The range of correlation of the B-spline copulas is examined, and the Fréchet-Hoeffding upper bound is proved to be attained when the number of B-spline functions goes to infinity. As the B-spline functions are well-known to be an ordercomplete weak Tchebycheff system from which the property of total positivity of any order $\left(\mathrm{TP}_{\infty}\right)$ follows for the maximum correlation case, the results given here extend classical results for the Bernstein copulas. In addition, we derive in terms of the Stirling numbers of the second kind an explicit formula for the moments of the related B-spline functions on $[0, \infty)$.
\end{abstract}

Keywords Bernstein copula • Fréchet-Hoeffding upper bound · Ordercomplete weak Tchebycheff system - Schur function - Stirling number of the second kind - Total positivity of order $r$

This work was supported by JSPS KAKENHI Grants, Numbers 16K00060 and 16H02792.

\author{
X. Dou \\ Waseda University, 3-4-1 Ohkubo, Shinjuku, Tokyo 169-8555, Japan \\ Tel.: +81-3-5286-2358 \\ E-mail: xiaoling@aoni.waseda.jp \\ S. Kuriki \\ The Institute of Statistical Mathematics, 10-3 Midoricho, Tachikawa, Tokyo 190-8562, Japan \\ G. D. Lin \\ Institute of Statistical Science, Academia Sinica, Taipei 11529, Taiwan, R.O.C. \\ D. Richards \\ Department of Statistics, Pennsylvania State University, University Park, PA 16802, U.S.A.
}




\section{Introduction: A review of the Bernstein copulas}

A novel method that applied the theory of order statistics to construct multivariate distributions with given marginal distributions was developed by Baker [1]. We refer to Lin, et al. [16] for a recent survey of this topic.

Baker's idea, applied to univariate cumulative distribution functions $F$ and $G$, can be described as follows: Let $\left\{X_{1}, \ldots, X_{n}\right\}$ and $\left\{Y_{1}, \ldots, Y_{n}\right\}$ be independent random samples from the distributions $F$ and $G$, respectively. Let $X_{k, n}$ be the $k$ th smallest order statistic of $\left\{X_{1}, \ldots, X_{n}\right\}$, and denote by $F_{k, n}$ the distribution of $X_{k, n}$; we write this as $X_{k, n} \sim F_{k, n}$. Similarly, we denote by $Y_{k, n}$ the $k$ th smallest order statistic of $\left\{Y_{1}, \ldots, Y_{n}\right\}$ and we let $G_{k, n}$ be its corresponding distribution, written $Y_{k, n} \sim G_{k, n}$. (Note that $F$ and $G$ can be discrete distributions.)

Let $R=\left(r_{k \ell}\right)_{1 \leq k, \ell \leq n}$ be a parameter matrix whose matrix entries $r_{k \ell}$ satisfy the conditions

$$
\sum_{k=1}^{n} r_{k \ell}=\sum_{\ell=1}^{n} r_{k \ell}=\frac{1}{n}, \quad r_{k \ell} \geq 0, k, \ell=1,2, \ldots, n .
$$

Now choose the pair $\left(X_{k, n}, Y_{\ell, n}\right)$ with probability $r_{k \ell}, k, \ell=1,2, \ldots, n$. Then $\left(X_{k, n}, Y_{\ell, n}\right)$ follows Baker's bivariate distribution: For $x, y \in \mathbb{R}$, the joint cumulative distribution function $H(x, y):=\operatorname{Pr}\left(X_{k, n} \leq x, Y_{k, n} \leq y\right)$ satisfies

$$
\begin{aligned}
H(x, y) & =\sum_{k=1}^{n} \sum_{\ell=1}^{n} r_{k \ell} F_{k, n}(x) G_{\ell, n}(y) \\
& =\left(F_{1, n}(x), \ldots, F_{n, n}(x)\right) R\left(G_{1, n}(y), \ldots, G_{n, n}(y)\right)^{\top},
\end{aligned}
$$

where "T" denotes transpose. It is immediately evident that $H$ has marginal distributions $F$ and $G$.

Let $B_{k, n}$ be the distribution function of the $k$ th smallest order statistic of a random sample of size $n$ from the uniform distribution $\mathcal{U}$ on $[0,1]$. It is well-known that

$$
B_{k, n}(u)=\int_{0}^{u} b_{k, n}(t) \mathrm{d} t
$$

where

$$
b_{k, n}(t)=n\left(\begin{array}{l}
n-1 \\
k-1
\end{array}\right) t^{k-1}(1-t)^{n-k},
$$

$t \in[0,1]$. Furthermore, $F_{k, n}$ equals the composition $B_{k, n} \circ F$ (see, e.g., Hwang and Lin [12]) and Baker's bivariate distribution (1.2) can be rewritten as

$$
H(x, y)=C(F(x), G(y) ; R),
$$

$x, y \in \mathbb{R}$, where, for $u, v \in[0,1]$,

$$
C(u, v ; R)=\sum_{k=1}^{n} \sum_{\ell=1}^{n} r_{k \ell} B_{k, n}(u) B_{\ell, n}(v)
$$


is a copula function with parameter matrix $R$ satisfying (1.1). Conversely, if the marginals $F$ and $G$ are equal to $\mathcal{U}$ then Baker's bivariate distribution (1.2) reduces to the copula $(1.4)$.

The copula $C(u, v ; R)$ in (1.4) is called the Bernstein copula with parameter matrix $R$ because $b_{k, n} / n$ is a Bernstein polynomial (see Dou, et al. [8]). By differentiating (1.4) with respect to $u$ and $v$, we obtain the Bernstein copula density:

$$
c(u, v ; R)=\sum_{k=1}^{n} \sum_{\ell=1}^{n} r_{k \ell} b_{k, n}(u) b_{\ell, n}(v)
$$

$u, v \in[0,1]$.

Within the parameter space (1.1) of $R$, the maximum correlation is attained when $r_{k \ell}=(1 / n) \delta_{k \ell}$, i.e., $R=(1 / n) I_{n}$ :

$$
C^{*}(u, v):=C\left(u, v ;(1 / n) I_{n}\right)=\frac{1}{n} \sum_{k=1}^{n} B_{k, n}(u) B_{k, n}(v),
$$

with corresponding density

$$
c^{*}(u, v):=c\left(u, v ;(1 / n) I_{n}\right)=\frac{1}{n} \sum_{k=1}^{n} b_{k, n}(u) b_{k, n}(v),
$$

$u, v \in[0,1]$.

Dou, et al. [7] proved that the maximum correlation copula $C^{*}(u, v)$ and its density $c^{*}(u, v)$ both are totally positive of order $2\left(\mathrm{TP}_{2}\right)$ in $(u, v)[14,13$, $22]$. One of the main purposes of the present paper is to show further that both $C^{*}$ and $c^{*}$ are $\mathrm{TP}_{\infty}$, i.e., $\mathrm{TP}_{r}$ for all $r \geq 2$.

In Section 2, we introduce first the general order-complete weak Tchebycheff (OCWT) systems and then a class of copulas, based on B-spline functions, that includes the Bernstein copulas $C(u, v ; R)$ in (1.4). The maximum correlation copula $C^{*}(u, v)$ and its total positivity properties are investigated in Sections 3 and 4, respectively. Finally, in Section 5 we calculate the moments of the related B-spline functions on $[0, \infty)$ and make the connection with the Stirling numbers of the second kind.

\section{B-spline copulas}

We consider first a general setting based on OCWT systems, and then we define a class of B-spline copulas that includes the Bernstein copulas as special cases. In other words, we will show that a larger class of candidate copulas can play the same roles as $B_{k, n}$ and $R$ while still retaining the desired properties of the copula $C(u, v ; R)$.

Let $q_{k} \geq 0, k=1, \ldots, n, \sum_{k=1}^{n} q_{k}=1$, and let $\phi_{1}, \ldots, \phi_{n}$ be probability densities on $[0,1]$ such that

$$
\sum_{k=1}^{n} q_{k} \phi_{k}(t)=1
$$


$t \in[0,1]$. We assume further that $\left\{\phi_{1}, \ldots, \phi_{n}\right\}$ is an order-complete weak Tchebycheff system (OCWT-system), i.e.,

(i) $\phi_{1}, \ldots, \phi_{n}$ are linearly independent, and

(ii) $\phi_{k}(t)$ is totally positive of order $n\left(T P_{n}\right)$ in $(k, t)$, i.e., for each $r=1, \ldots, n$,

$$
\operatorname{det}\left(\phi_{k_{i}}\left(t_{j}\right)\right)_{r \times r} \geq 0
$$

for all $k_{1}>\cdots>k_{r}$ and $t_{1}>\cdots>t_{r}$.

See Karlin and Studden [14, Chapter 1] or Schumaker [23, Chapter 2] for examples of OCWT systems.

Let $q_{1 k} \geq 0, k=1, \ldots, n_{1}$, such that $\sum_{k=1}^{n_{1}} q_{1 k}=1$. Also, let $q_{2 \ell} \geq 0$, $\ell=1, \ldots, n_{2}$, such that $\sum_{\ell=1}^{n_{2}} q_{2 \ell}=1$. Letting

$$
\Phi_{k}(u)=\int_{0}^{u} \phi_{k}(t) \mathrm{d} t
$$

$u \in[0,1]$, we define the B-spline copula, a generalization of the Bernstein copula (1.4), by

$$
C(u, v ; R)=\sum_{k=1}^{n_{1}} \sum_{\ell=1}^{n_{2}} r_{k \ell} \Phi_{k}(u) \Phi_{\ell}(v)
$$

$u, v \in[0,1]$, with parameter matrix

$$
\begin{aligned}
& R=\left(r_{k \ell}\right)_{1 \leq k \leq n_{1} ; 1 \leq \ell \leq n_{2}}, \quad r_{k \ell} \geq 0 \\
& \sum_{k=1}^{n_{1}} r_{k \ell}=q_{2 \ell}, \sum_{\ell=1}^{n_{2}} r_{k \ell}=q_{1 k}, \quad k=1,2, \ldots, n_{1}, \quad \ell=1,2, \ldots, n_{2} .
\end{aligned}
$$

The copula (2.3) is a bona fide copula since, for any $u \in[0,1]$,

$$
\begin{aligned}
C(u, 1 ; R) & =\sum_{k=1}^{n_{1}} \sum_{\ell=1}^{n_{2}} r_{k \ell} \Phi_{k}(u)=\sum_{k=1}^{n_{1}} q_{1 k} \Phi_{k}(u) \\
& =\int_{0}^{u} \sum_{k=1}^{n_{1}} q_{1 k} \phi_{k}(t) \mathrm{d} t=\int_{0}^{u} 1 \mathrm{~d} t=u ;
\end{aligned}
$$

and similarly, $C(1, v ; R)=v, v \in[0,1]$.

Throughout the paper, we restrict our attention to the case in which $n_{1}=$ $n_{2}=n$ and $q_{1 k}=q_{2 k}=q_{k}$; further, we use the notation $Q=\operatorname{diag}\left(q_{k}\right)_{1 \leq k \leq n}$ for the diagonal matrix with diagonal entries $q_{1}, \ldots, q_{n}$.

Theorem 1 For the copula (2.3) with the parameter space (2.4), the maximum correlation is attained when $r_{k \ell}=q_{k} \delta_{k \ell}$, equivalently, $R=Q$. 
In the maximum correlation case, $C(u, v ; R)$ becomes

$$
C^{*}(u, v):=C(u, v ; Q)=\sum_{k=1}^{n} q_{k} \Phi_{k}(u) \Phi_{k}(v),
$$

$u, v \in[0,1]$.

To prove Theorem 1, we need the following crucial lemma. This result is a generalization of the weak majorization inequality on the closed simplicial cone

$$
\mathcal{D}_{+}=\left\{\left(x_{1}, \ldots, x_{n}\right): x_{1} \geq \cdots \geq x_{n} \geq 0\right\} \subset \mathbb{R}_{+}^{n}
$$

for doubly stochastic matrices [19, p. 639].

Lemma 1 Let $a_{1} \geq \cdots \geq a_{n} \geq 0$ and $b_{1} \geq \cdots \geq b_{n} \geq 0$ be given. Let $q_{1}, \ldots, q_{n} \geq 0$ satisfy $\sum_{k=1}^{n} q_{k}=1$. Then,

$$
\max _{\substack{\sum_{k} r_{k \ell}=q_{\ell} \\ \sum_{\ell} r_{k \ell}=q_{k} \\ r_{k \ell} \geq 0}} \sum_{k=1}^{n} \sum_{\ell=1}^{n} r_{k \ell} a_{k} b_{\ell}=\sum_{k=1}^{n} q_{k} a_{k} b_{k} .
$$

Proof. Let $\mathbf{a}=\left(a_{1}, \ldots, a_{n}\right)$ and $\mathbf{b}=\left(b_{1}, \ldots, b_{n}\right)$. Define

$$
p_{k \ell}= \begin{cases}r_{k \ell}, & k \neq \ell, \\ r_{k k}+1-q_{k}, & k=l .\end{cases}
$$

For given $Q=\operatorname{diag}\left(q_{k}\right)_{1 \leq k \leq n}$, it is straightforward to see that $P=R+I_{n}-Q$ is a $n \times n$ doubly stochastic matrix. Hence, by the famous characterization of majorization due to Hardy, Littlewood, and Pólya [10], the vector $\mathbf{c}=\mathbf{a} P$ is majorized by $\mathbf{a}$, denoted $\mathbf{c} \prec \mathbf{a}$.

We now rearrange the components of $\mathbf{c}=\left(c_{1}, \ldots, c_{n}\right)$ in decreasing order, listing them as $c_{[1]} \geq \cdots \geq c_{[n]}$, and let $\mathbf{c}^{*}=\left(c_{[1]}, \ldots, c_{[n]}\right)$. Then we have also $\mathbf{c}^{*} \prec \mathbf{a}$ and hence $\mathbf{c}^{*} \mathbf{b}^{\top} \leq \mathbf{a} \mathbf{b}^{\top}$ because $\mathbf{a}, \mathbf{b}, \mathbf{c}^{*} \in \mathcal{D}_{+}$(see [19, p. 133]). On the other hand, note that $\mathbf{c b}^{\top} \leq \mathbf{c}^{*} \mathbf{b}^{\top}$ because $\mathbf{b}, \mathbf{c}^{*} \in \mathcal{D}_{+}$. These results together imply that $\mathbf{a} P \mathbf{b}^{\top}=\mathbf{c b}^{\top} \leq \mathbf{c}^{*} \mathbf{b}^{\top} \leq \mathbf{a} \mathbf{b}^{\top}$.

Consequently, $\max _{P} \mathbf{a} P \mathbf{b}^{\top}=\mathbf{a} \mathbf{b}^{\top}$, which we can write alternatively as

$$
\max _{\substack{\sum_{k} p_{k \ell}=1 \\ \sum_{\ell} p_{k \ell}=1 \\ p_{k \ell} \geq 0}} \sum_{k=1}^{n} \sum_{\ell=1}^{n} p_{k \ell} a_{k} b_{\ell}=\sum_{k=1}^{n} a_{k} b_{k} .
$$

Equivalently, $\max _{R} \mathbf{a} R \mathbf{b}^{\top}=\mathbf{a} Q \mathbf{b}^{\top}$ by cancelling the common term $\mathbf{a b}^{\top}$ on both sides above. This is exactly (2.6), so the proof now is complete.

Proof of Theorem 1. Since $\left\{\phi_{1}, \ldots, \phi_{n}\right\}$ is an OCWT-system then, for all $i<j$ and $s<t$,

$$
\phi_{i}(s) \phi_{j}(t)-\phi_{j}(s) \phi_{i}(t)=\operatorname{det}\left(\begin{array}{cc}
\phi_{i}(s) & \phi_{i}(t) \\
\phi_{j}(s) & \phi_{j}(t)
\end{array}\right) \geq 0
$$


Integrating this inequality with respect to $(s, t)$ over $s \in(0, u)$ and $t \in(u, 1)$, we obtain

$$
\Phi_{i}(u)\left(1-\Phi_{j}(u)\right)-\Phi_{j}(u)\left(1-\Phi_{i}(u)\right)=\Phi_{i}(u)-\Phi_{j}(u) \geq 0,
$$

$u \in[0,1]$. Therefore, we obtain the stochastic order,

$$
\Phi_{1}(u) \geq \Phi_{2}(u) \geq \cdots \geq \Phi_{k}(u)
$$

for all $u \in[0,1]$. Combining this result with Lemma 1 , we obtain the inequality

$$
C^{*}(u, v):=C(u, v ; Q) \geq C(u, v ; R)
$$

for all $u, v \in[0,1]$ and $R$ satisfying (2.4). The theorem now follows from Hoeffding's covariance formula,

$$
\operatorname{Cov}(X, Y)=\int_{-\infty}^{\infty} \int_{-\infty}^{\infty}[\operatorname{Pr}(X \leq x, Y \leq y)-\operatorname{Pr}(X \leq x) \operatorname{Pr}(Y \leq y)] \mathrm{d} x \mathrm{~d} y
$$

(see, e.g., [16]). The proof is complete.

Functions $\phi_{k}$ satisfying (2.1) and (2.2) can be constructed by B-spline functions as we now show. Let $N_{i}^{d}$ be a B-spline function on $[0,1]$ of degree $d(\geq 0)$ defined as a non-zero B-spline basis with $m+2 d+1$ knots:

$$
\underbrace{t_{-d}=\cdots=t_{-1}}_{d}=t_{0}=0<t_{1}<\cdots<t_{m-1}<1=t_{m}=\underbrace{t_{m+1}=\cdots=t_{m+d}}_{d} .
$$

Then, $N_{i}^{d}(t)$ is generated by the recursion formula,

$$
\begin{aligned}
N_{i}^{d}(t) & =\frac{t-t_{i}}{t_{i+d}-t_{i}} N_{i}^{d-1}(t)+\frac{t_{i+d+1}-t}{t_{i+d+1}-t_{i+1}} N_{i+1}^{d-1}(t) \\
& =\frac{t-t_{i}}{t_{i+d}-t_{i}} N_{i}^{d-1}(t)+\left(1-\frac{t-t_{i+1}}{t_{i+d+1}-t_{i+1}}\right) N_{i+1}^{d-1}(t),
\end{aligned}
$$

$t \in[0,1]$, for $i=-d, \ldots,-1,0,1, \ldots, m-1$, with initial conditions

$$
N_{i}^{0}(t)= \begin{cases}1, & i<m \text { and } t \in\left[t_{i}, t_{i+1}\right), \\ & \text { or } i=m-1 \text { and } t=t_{m}=1, \\ 0, & \text { otherwise }\end{cases}
$$

(see $[4,6,21])$. The number of non-zero bases is

$$
n=m+d \text {. }
$$

The B-spline is known to satisfy

(i) $N_{i}^{d}(t) \geq 0, t \in[0,1]$, 
(ii) The support is given by

$$
\operatorname{supp} N_{i}^{d}=\overline{\left\{t \mid N_{i}^{d}(t)>0\right\}}=\left[t_{i}, t_{i+d+1}\right],
$$

$i=-d, \ldots,-1,0,1, \ldots, m-1$, and

(iii) The "partition of unity" property:

$$
\sum_{i=-d}^{m-1} N_{i}^{d}(t)=1 \text { for all } t \in[0,1]
$$

For given $d$ and $m$, let

$$
q_{k}=q_{k, d}=\int_{0}^{1} N_{k-d-1}^{d}(t) \mathrm{d} t, \quad \phi_{k}(t)=\phi_{k, d}(t)=\frac{1}{q_{k}} N_{k-d-1}^{d}(t),
$$

where $t \in[0,1]$ and $k=1,2, \ldots, n(=m+d)$. Then, $(2.1)$ holds, and we have the following result (see [5], or [23, Theorems 4.18 and 4.65]).

Theorem 2 Under the hypotheses (2.7) and (2.8), the set $\left\{N_{i}^{d}\right\}_{i=-d}^{m-1}$ of Bspline functions, and hence also the B-spline system $\left\{\phi_{1}, \ldots, \phi_{n}\right\}$, forms an OCWT-system satisfying (2.2).

To illustrate the use of B-spline systems, we now provide some examples.

Theorem 3 Let $m=1$ and the degree $d=n-1(=n-m)$. Then the B-splines (2.8) reduce to the Bernstein system (1.3). Specifically, for $k=1, \ldots, n$ and $t \in[0,1]$

$$
q_{k}=q_{k, d}=\frac{1}{n}, \quad \phi_{k}(t)=\phi_{k, d}(t)=b_{k, n}(t)
$$

Proof. We prove the result by induction on $d$. Note that for $d=0$ (i.e., $n=1$ ), $N_{0}^{0}(t)=1, t \in[0,1]$, and hence

$$
q_{1}=q_{1,0}=\int_{0}^{1} N_{0}^{0}(t) \mathrm{d} t=1, \quad \phi_{1}(t)=\phi_{1,0}(t)=N_{0}^{0}(t)=b_{1,1}(t),
$$

$t \in[0,1]$. For $d=1$ (i.e., $n=2$ ), we have the required $N_{i}^{1}, q_{k, 1}$, and $\phi_{k, 1}$ as follows:

$$
\begin{gathered}
N_{-1}^{1}(t)=(1-t) N_{0}^{0}(t)=1-t, \quad N_{0}^{1}(t)=t N_{0}^{0}(t)=t, \quad t \in[0,1] \\
q_{1}=q_{1,1}=\int_{0}^{1} N_{-1}^{1}(t) \mathrm{d} t=1 / 2, \quad q_{2}=q_{2,1}=\int_{0}^{1} N_{0}^{1}(t) \mathrm{d} t=1 / 2, \\
\phi_{1}(t)=\phi_{1,1}(t)=2 N_{-1}^{1}(t)=b_{1,2}(t) \\
\phi_{2}(t)=\phi_{2,1}(t)=2 N_{0}^{1}(t)=b_{2,2}(t), \quad t \in[0,1] .
\end{gathered}
$$


Assume that the theorem holds true for $d=n-2$, then we want to prove $(2.9)$ for $d=n-1$. In this case, the B-spline functions are of the form

$$
N_{k-n}^{n-1}(t)= \begin{cases}(1-t) N_{2-n}^{n-2}(t)=(n-1)^{-1}(1-t) b_{1, n-1}(t), & k=1, \\ t N_{k-n}^{n-2}(t)+(1-t) N_{k-n+1}^{n-2}(t) & \\ \quad=(n-1)^{-1}\left[t b_{k-1, n-1}(t)+(1-t) b_{k, n-1}(t)\right], & 1<k<n, \\ t N_{0}^{n-2}(t)=(n-1)^{-1} t b_{n-1, n-1}(t), & k=n .\end{cases}
$$

It can be shown that for $k=1,2, \ldots, n$,

$$
q_{k}=q_{k, n-1}=\int_{0}^{1} N_{k-n}^{n-1}(t) \mathrm{d} t=1 / n
$$

and

$$
N_{k-n}^{n-1}(t)=q_{k} b_{k, n}(t),
$$

$t \in[0,1]$. This completes the proof.

From now on, for simplicity, we consider only the B-spline with equallyspaced knots, i.e., the B-spline functions on $[0,1]$ of order $d$ having knots given in (2.7) with $t_{i}=i / m, i=1,2, \ldots, m-1$.

Example 1 Suppose $d=0$, i.e., $n=m$; then the B-spline system becomes a "histogram". Namely, for $k=1,2, \ldots, n$,

$$
q_{k}=q_{k, 0}=\frac{1}{n}, \quad \phi_{k}(t)=\phi_{k, 0}(t)=n \mathbb{1}_{\left[\frac{k-1}{n}, \frac{k}{n}\right)}(t),
$$

$t \in[0,1]$, where $\mathbb{1}_{A}$ denotes the indicator function of the set $A$.

Example 2 For $d=1$, i.e., $n=m+1$, we have

$$
q_{1}=q_{1,1}=\frac{1}{2 m}, \quad q_{2}=q_{3}=\cdots=q_{m-1}=q_{m}=\frac{1}{m}, \quad q_{n}=q_{n, 1}=\frac{1}{2 m},
$$

and the B-spline system is

$$
\begin{aligned}
& \phi_{1}(t)=\phi_{1,1}(t)=q_{1}^{-1} N_{-1}^{1}(t)=2 m(1-m t) \mathbb{1}_{\left[0, \frac{1}{m}\right)}(t), \\
& \phi_{n}(t)=\phi_{n, 1}(t)=q_{n}^{-1} N_{m-1}^{1}(t)=2 m(m t-m+1) \mathbb{1}_{\left[1-\frac{1}{m}, 1\right)}(t),
\end{aligned}
$$

and, for $k=2,3, \ldots, n-1$,

$$
\begin{aligned}
\phi_{k}(t)=\phi_{k, 1}(t) & =q_{k}^{-1} N_{k-2}^{1}(t) \\
& =q_{k}^{-1}\left[(m t-k+2) N_{k-2}^{0}(t)+(k-m t) N_{k-1}^{0}(t)\right] \\
& =m\left[(m t-k+2) \mathbb{1}_{\left[\frac{k-2}{m}, \frac{k-1}{m}\right)}(t)+(k-m t) \mathbb{1}_{\left[\frac{k-1}{m}, \frac{k}{m}\right)}(t)\right],
\end{aligned}
$$

$t \in[0,1]$.

We remark that Shen, et al. [24] earlier proposed the "linear B-spline copula", which corresponds to the case $d=1$. 
Example 3 For $d=3$ and $m=2$, i.e., $n=5$, we have

$$
q_{1}=1 / 8, \quad q_{2}=q_{3}=q_{4}=1 / 4, \quad q_{5}=1 / 8,
$$

and the B-spline system is

$$
\begin{aligned}
& \phi_{1}(t)=q_{1}^{-1} N_{-3}^{3}(t)=8(1-2 t)^{3} \mathbb{1}_{\left[0, \frac{1}{2}\right)}(t), \\
& \phi_{2}(t)=q_{2}^{-1} N_{-2}^{3}(t)=8 t\left(7 t^{2}-9 t+3\right) \mathbb{1}_{\left[0, \frac{1}{2}\right)}(t)+8(1-t)^{3} \mathbb{1}_{\left[\frac{1}{2}, 1\right)}(t), \\
& \phi_{3}(t)=q_{3}^{-1} N_{-1}^{3}(t)=8 t^{2}(3-4 t) \mathbb{1}_{\left[0, \frac{1}{2}\right)}(t)+8(1-t)^{2}(4 t-1) \mathbb{1}_{\left[\frac{1}{2}, 1\right)}(t), \\
& \phi_{4}(t)=\phi_{2}(1-t), \\
& \phi_{5}(t)=\phi_{1}(1-t),
\end{aligned}
$$

$t \in[0,1]$. The means of the densities $\phi_{1}, \ldots, \phi_{5}$ are $1 / 10,3 / 10,1 / 2,7 / 10$, and $9 / 10$, respectively. We use these values in the computation of Table 1 of maximum correlations for $(n, d)=(5,3)$.

\section{The maximum correlation copula: Range of correlation}

For copula functions, the range of the correlation is of particular importance. In particular, great attention is paid to the maximum achievable correlation (see, e.g., Lin and Huang [17]). By Theorem 1, the maximum is attained when the copula density is

$$
c^{*}(u, v)=\sum_{k=1}^{n} q_{k} \phi_{k}(u) \phi_{k}(v), \quad u, v \in[0,1] .
$$

Suppose that $(U, V)$ is from the copula density (3.1). Then,

$$
E[U V]=\sum_{k=1}^{n} q_{k}\left(\int_{0}^{1} u \phi_{k}(u) \mathrm{d} u\right)^{2} .
$$

Noting that $E[U]=E[V]=1 / 2$ and $\operatorname{Var}(U)=\operatorname{Var}(V)=1 / 12$, it follows that

$$
\operatorname{corr}(U, V)=12\left(E[U V]-\frac{1}{4}\right)
$$

In the Bernstein case $(m=1)$, it follows from Theorem 3 that $E[U V]=$ $(2 n+1) / 6(n+1)$ and hence

$$
\operatorname{corr}(U, V)=1-\frac{2}{n+1} .
$$

In the case of the B-spline of order zero, given in (2.10),

$$
\operatorname{corr}(U, V)=12\left(\frac{1}{n} \sum_{k=1}^{n}\left(n \int_{(k-1) / n}^{k / n} t \mathrm{~d} t\right)^{2}-\frac{1}{4}\right)=1-\frac{1}{n^{2}}
$$


In particular, when $d=0$ and $n=m=1, \phi_{1}(t)=1$ on $[0,1]$, and hence $C^{*}(u, v)=0, u, v \in[0,1]$. This is the independent case, so $\operatorname{corr}(U, V)=0$.

In order to calculate the maximum correlation for general $d$, we present first a lemma in which it is understood that the vectors $\left(q_{k}\right)$ and $\left(r_{k}\right)$ reduce to the central parts when $d=0$.

Lemma 2 Suppose that $m \geq d \geq 0$, i.e., $n=m+d \geq 2 d \geq 0$. Let $N_{i}^{d}$, $i=-d,-d+1, \ldots, m-1$, be the B-spline functions on $[0,1]$ of order $d$ having knots (2.7) with $t_{i}=i / m, i=0,1, \ldots, m$. In addition, denote the integral and the first moment of $N_{k-d-1}^{d}$ by

$$
q_{k}=\int_{0}^{1} N_{k-d-1}^{d}(t) \mathrm{d} t \quad \text { and } \quad r_{k}=\int_{0}^{1} t N_{k-d-1}^{d}(t) \mathrm{d} t
$$

$k=1, \ldots, n$. Then,

$$
\begin{aligned}
\left(q_{k}\right)_{1 \leq k \leq n}= & \frac{1}{m}(\underbrace{}_{\left(r_{k}\right)_{1 \leq k \leq n}=\frac{1}{m^{2}}}(\underbrace{\frac{1}{d+1}, \frac{2}{d+1}, \ldots, \frac{d}{d+1}, \underbrace{\frac{1^{2}(1+1)}{2(d+1)(d+2)}, \frac{2^{2}(2+1)}{2(d+1)(d+2)}, \ldots, \frac{d^{2}(d+1)}{2(d+1)(d+2)}}_{m-d}, \underbrace{\left.\frac{d}{d+1}, \frac{d-1}{d+1}, \ldots, \frac{1}{d+1}\right)}_{d}}_{d} \\
& \underbrace{\frac{d+1}{2}, \frac{d+3}{2}, \ldots, \frac{2 m-1-d}{2}}_{m-d}, \underbrace{m^{2}\left(q_{d}-r_{d}\right), \ldots, m^{2}\left(q_{1}-r_{1}\right)}_{d}) .
\end{aligned}
$$

Proof. For $1 \leq k \leq m$, we have $q_{k}=\gamma_{k-d-1}^{d}(0) / m$ and $r_{k}=\gamma_{k-d-1}^{d}(1) / m^{2}$, where $\gamma_{i}^{d}(0)$ and $\gamma_{i}^{d}(1)$ are given below in (5.8) and (5.9), respectively. Also, for $k=m+1, \ldots, m+d=n$, we have the relations

$$
q_{k}=q_{n+1-k}, \quad r_{k}=q_{n+1-k}-r_{n+1-k},
$$

because $N_{k-d-1}^{d}(t)=N_{m-k}^{d}(1-t)=N_{(n+1-k)-d-1}^{d}(1-t), t \in[0,1]$. Solving the equations (3.4) in a successive manner, we obtain the stated results.

Theorem 4 Under the assumptions of Lemma 2, suppose that $(U, V)$ have the copula density $c^{*}$ in (3.1) with $\phi_{k}$ defined through the B-spline functions (2.8) having knots given in Lemma 2. Then the correlation of $(U, V)$ is

$$
\operatorname{corr}(U, V)=1-\frac{d+1}{(n-d)^{2}}+\frac{d(d+3)(2 d+3)}{5(d+2)(n-d)^{3}} .
$$


Proof. Using (3.2), (3.4), and the notations in Lemma 2, write first

$$
\begin{aligned}
E[U V]=\sum_{k=1}^{n} \frac{r_{k}^{2}}{q_{k}} & =\sum_{k=1}^{m} \frac{r_{k}^{2}}{q_{k}}+\sum_{k=1}^{d} \frac{\left(q_{k}-r_{k}\right)^{2}}{q_{k}} \\
& =\sum_{k=1}^{m} \frac{r_{k}^{2}}{q_{k}}+\sum_{k=1}^{d}\left(q_{k}-2 r_{k}+\frac{r_{k}^{2}}{q_{k}}\right) \\
& =2 \sum_{k=1}^{d} \frac{r_{k}^{2}}{q_{k}}+\sum_{k=1}^{d} q_{k}-2 \sum_{k=1}^{d} r_{k}+\sum_{k=d+1}^{m} \frac{r_{k}^{2}}{q_{k}} .
\end{aligned}
$$

The final result is obtained by substituting (3.6) in (3.3) and carry out the calculations to obtain (3.5) with the help of Lemma 2.

The maximum correlation in (3.5) remains valid for all cases $m \geq d \geq 0$. Further, the maximum correlation converges to 1 as $n \rightarrow \infty$, so we obtain $\operatorname{Var}(U-V)=(1-\operatorname{corr}(U, V)) / 6 \rightarrow 0$, or $V-U \rightarrow 0$ in probability. Therefore, as $n \rightarrow \infty$, the random variable $(U, V)=(U, U+(V-U))$ converges in law to $(U, U)$, a bivariate random variable whose joint distribution, remarkably, happens to provide the Fréchet-Hoeffding upper bound, $\min \{u, v\}$. Thus, we have the following result.

Theorem 5 Let $C^{*}$ be the maximum correlation copula function (2.5) that is constructed by the B-spline

$$
\left\{N_{k-d-1}^{d}\right\}_{k=1}^{n}=\left\{N_{i}^{d}: i=-d,-d+1, \ldots, m-2, m-1\right\}
$$

on $[0,1]$ of degree $d \geq 0$, having equally-spaced knots (2.7) with $t_{i}=i / m$, $i=0,1, \ldots, m$, where $m \geq d$. As $m \rightarrow \infty, C^{*}(u, v) \rightarrow \min \{u, v\}$ for all $u, v$, the Fréchet-Hoeffding upper bound.

Table 1 shows the maximum correlations when the number of basis functions is $n$. In view of Table 1 , the range of correlation for the B-spline copulas of small order $d$ is wider than that of the Bernstein copula. Indeed,

$$
\operatorname{corr}(U, V) \approx 1-\frac{d+1}{n^{2}} .
$$

On the other hand, $d$ determines the smoothness of the copula density. Consequently, some criterion is needed to evaluate data fitness so as to balance the accuracy of the approximation with the smoothness of the density; this problem will be studied in future work.

We conjecture that Theorem 5 holds in more general settings.

Conjecture 1 Let $(U, V)$ be distributed as the maximum correlation distribution (2.5) constructed by the B-spline

$$
\left\{N_{k-d-1}^{d}\right\}_{k=1}^{n}=\left\{N_{i}^{d}: i=-d,-d+1, \ldots, m-2, m-1\right\}
$$

on $[0,1]$, of degree $d \geq 0$, with the knots (2.7). As $m \rightarrow \infty$ with $\max _{1 \leq i \leq m} \mid t_{i-1}-$ $t_{i} \mid \rightarrow 0, \operatorname{corr}(U, V)$ converges to 1 ; hence, for all $u, v, C^{*}(u, v)$ converges to $\min \{u, v\}$, the Fréchet-Hoeffding upper bound. 
Table 1 Maximum correlations

\begin{tabular}{c|ccccc}
\hline & Bernstein* & $d=0$ & $d=1$ & $d=2$ & $d=3$ \\
\hline$n=2$ & 0.333 & 0.75 & 0.333 & NA & NA \\
$n=3$ & 0.5 & 0.889 & 0.667 & $0.5^{*}$ & NA \\
$n=4$ & 0.6 & 0.938 & 0.827 & 0.688 & $0.6^{*}$ \\
$n=5$ & 0.667 & 0.96 & 0.896 & 0.796 & $0.72^{* *}$ \\
$n=6$ & 0.714 & 0.972 & 0.931 & 0.867 & 0.796 \\
$n=7$ & 0.75 & 0.980 & 0.951 & 0.908 & 0.851 \\
$n=8$ & 0.778 & 0.984 & 0.963 & 0.933 & 0.892 \\
$n=9$ & 0.8 & 0.988 & 0.971 & 0.949 & 0.919 \\
$n=10$ & 0.818 & 0.99 & 0.977 & 0.960 & 0.937 \\
\hline$n$ & $1-\frac{1}{n+1}$ & $1-\frac{1}{n^{2}}$ & $1-\frac{2(3 n-5)}{3(n-1)^{3}}$ & $1-\frac{6 n-19}{2(n-2)^{3}}$ & $1-\frac{2(50 n-231)}{25(n-3)^{3}}$ \\
\hline
\end{tabular}

\section{The maximum correlation copula: Total positivity}

The next two results improve significantly the previous ones about the Bernstein copulas.

Theorem 6 The copula $C^{*}$ in (2.5) is $T P_{\infty}$, i.e., for any $r \geq 1$,

$$
\operatorname{det}\left(C^{*}\left(u_{i}, v_{j}\right)\right)_{r \times r} \geq 0
$$

for all $u_{1}>\cdots>u_{r}$ and $v_{1}>\cdots>v_{r}$.

Proof. All determinants arising in the proof are of order $r$, unless otherwise specified. Further, we consider two cases: (I) $r>n$, and (II) $r \leq n$.

Case I: $r>n$. In this case, the $r \times r$ matrix $\left(C^{*}\left(u_{i}, v_{j}\right)\right)_{1 \leq i, j \leq r}$ satisfies

$$
\begin{aligned}
\left(C^{*}\left(u_{i}, v_{j}\right)\right)_{1 \leq i, j \leq r} & =\left(\sum_{k=1}^{n} q_{k} \Phi_{k}\left(u_{i}\right) \Phi_{k}\left(v_{j}\right)\right)_{1 \leq i, j \leq r} \\
& =\left(q_{j} \Phi_{j}\left(u_{i}\right)\right)_{1 \leq i \leq r ; 1 \leq j \leq n}\left(\Phi_{i}\left(v_{j}\right)\right)_{1 \leq i \leq n ; 1 \leq j \leq r} .
\end{aligned}
$$

Consequently, the rank of this matrix is at most $n$, and hence is degenerate. Therefore, it follows obviously that $\operatorname{det}\left(C^{*}\left(u_{i}, v_{j}\right)\right)=0$.

Case II: $r \leq n$. We will show that $\operatorname{det}\left(C^{*}\left(u_{i}, v_{j}\right)\right) \geq 0$. By the BinetCauchy formula,

$$
\begin{aligned}
\operatorname{det}\left(C^{*}\left(u_{i}, v_{j}\right)\right) & =\operatorname{det}\left(\sum_{k=1}^{n} q_{k} \Phi_{k}\left(u_{i}\right) \Phi_{k}\left(v_{j}\right)\right) \\
& =\sum_{n \geq k_{1}>\cdots>k_{r} \geq 1} \cdots \sum_{i=1}\left(\prod_{i=1}^{r} q_{k_{i}}\right) \operatorname{det}\left(\Phi_{k_{i}}\left(u_{j}\right)\right) \operatorname{det}\left(\Phi_{k_{i}}\left(v_{j}\right)\right) .
\end{aligned}
$$


Writing

$$
\operatorname{det}\left(\Phi_{k_{i}}\left(u_{j}\right)\right)=\operatorname{det}\left(\int_{0}^{u_{j}} \phi_{k_{i}}(t) \mathrm{d} t\right)=\operatorname{det}\left(\int_{0}^{1} \phi_{k_{i}}(t) \mathbb{1}_{\left(0, u_{j}\right)}(t) \mathrm{d} t\right),
$$

it follows by the continuous version of the Binet-Cauchy formula $[9,13]$ that

$$
\begin{aligned}
\operatorname{det}\left(\int_{0}^{1} \phi_{k_{i}}(t) \mathbb{1}_{\left(0, u_{j}\right)}(t) \mathrm{d} t\right) & \\
= & \int_{1>t_{1}>\cdots>t_{r}>0} \cdots \int_{i=1} \operatorname{det}\left(\phi_{k_{j}}\left(t_{i}\right)\right) \operatorname{det}\left(\mathbb{1}_{\left(0, u_{i}\right)}\left(t_{j}\right)\right) \prod_{i}^{r} \mathrm{~d} t_{i}
\end{aligned}
$$

By Theorem 2, $\left\{\phi_{k_{1}}, \ldots, \phi_{k_{r}}\right\}$ is an OCWT-system, hence

$$
\operatorname{det}\left(\phi_{k_{j}}\left(t_{i}\right)\right) \geq 0
$$

for all $k_{1}>\cdots>k_{r}$ and $t_{1}>\cdots>t_{r}$. Also, it is well-known from $[13,14]$ that

$$
\operatorname{det}\left(\mathbb{1}_{\left(0, u_{i}\right)}\left(t_{j}\right)\right) \geq 0
$$

for all $u_{1}>\cdots>u_{r}$ and $t_{1}>\cdots>t_{r}$.

Therefore, we deduce from (4.2) and (4.3) that $\operatorname{det}\left(\Phi_{k_{i}}\left(u_{j}\right)\right) \geq 0$ for all $k_{1}>\cdots>k_{r}$ and $u_{1}>\cdots>u_{r}$. Similarly, we obtain $\operatorname{det}\left(\Phi_{k_{i}}\left(v_{j}\right)\right) \geq 0$ for $k_{1}>\cdots>k_{r}$ and $v_{1}>\cdots>v_{r}$. Hence, it follows from (4.1) that $\operatorname{det}\left(C^{*}\left(u_{i}, v_{j}\right)\right) \geq 0$ for $u_{1}>\cdots>u_{r}$ and $v_{1}>\cdots>v_{r}$. The proof is complete.

Remark 1 For the case of the Bernstein copula, we note that (4.4) is proved as follows. Consider

$$
\phi_{k}(t)=b_{k, n}(t)=k\left(\begin{array}{l}
n \\
k
\end{array}\right) t^{k-1}(1-t)^{n-k}
$$

$t \in[0,1]$. Then,

$$
\operatorname{det}\left(\phi_{k_{j}}\left(t_{i}\right)\right)=\left(\prod_{i=1}^{r} k_{i}\left(\begin{array}{c}
n \\
k_{i}
\end{array}\right)\right) \operatorname{det}\left(t_{i}^{k_{j}-1}\left(1-t_{i}\right)^{n-k_{j}}\right)
$$

and

$$
\begin{aligned}
\operatorname{det}\left(t_{i}^{k_{j}-1}\left(1-t_{i}\right)^{n-k_{j}}\right) & =\operatorname{det}\left(\left(\frac{t_{i}}{1-t_{i}}\right)^{k_{j}-1}\left(1-t_{i}\right)^{n-1}\right) \\
& =\prod_{i=1}^{r}\left(1-t_{i}\right)^{n-1} \cdot \operatorname{det}\left(\left(\frac{t_{i}}{1-t_{i}}\right)^{k_{j}-1}\right) .
\end{aligned}
$$

For $k_{1}>\cdots>k_{r} \geq 1$, set $k_{j}-1=\kappa_{j}+r-j, j=1, \ldots, r$, and define the partition $\kappa=\left(\kappa_{1}, \ldots, \kappa_{r}\right)$, i.e., $\kappa_{1}, \ldots, \kappa_{r}$ are nonnegative integers and $\kappa_{1} \geq \cdots \geq \kappa_{r}$. Also, let $z_{i}=t_{i} /\left(1-t_{i}\right), i=1, \ldots, r$, and let $z=\left(z_{1}, \ldots, z_{r}\right)$. 
Recall from [18] that the Schur function corresponding to the partition $\kappa$ is defined as

$$
\chi_{\kappa}(z)=\frac{\operatorname{det}\left(z_{i}^{k_{j}-1}\right)}{\prod_{i<j}\left(z_{i}-z_{j}\right)} .
$$

Then we obtain

$$
\begin{aligned}
\operatorname{det}\left(z_{i}^{k_{j}-1}\right) & =\chi_{\kappa}(z) \cdot \prod_{i<j}\left(z_{i}-z_{j}\right) \\
& =\chi_{\kappa}(z) \cdot \prod_{i<j}\left(\frac{t_{i}}{1-t_{i}}-\frac{t_{j}}{1-t_{j}}\right) \\
& =\chi_{\kappa}(z) \cdot \prod_{i<j} \frac{t_{i}-t_{j}}{\left(1-t_{i}\right)\left(1-t_{j}\right)} .
\end{aligned}
$$

It is well-known that $\chi_{\kappa}(z) \geq 0$ for and $z_{1}, \ldots, z_{r} \geq 0[9,18]$, and hence

$$
\operatorname{det}\left(t_{i}^{k_{j}-1}\left(1-t_{i}\right)^{n-k_{j}}\right) \geq 0
$$

for all $k_{1}>\cdots>k_{r}$ and $t_{1}>\cdots>t_{r}$. This completes the proof of (4.4).

As a consequence of Theorem 6, we obtain a new proof of the total positivity of the function $\min \{u, v\}$; see [13, Chapter 2].

Corollary 1 The Fréchet-Hoeffding upper bound, $\min \{u, v\}$, is $T P_{\infty}$.

Proof. Recall that for the Bernstein copula, $C^{*}(u, v)$ increases to $\min \{u, v\}$ as the number $n$ of basis functions goes to infinity (see Huang, et al. [11]). Moreover, it follows from Theorem 5 that for the equally-spaced knot B-spline copula, $C^{*}(u, v)$ converges to $\min \{u, v\}$. In either case, by taking the limit, as $n \rightarrow \infty$, of the $r \times r$ nonnegative determinant, $\operatorname{det}\left(C^{*}\left(u_{i}, v_{j}\right)\right)$, we obtain

$$
\min \{u, v\}=\lim _{n \rightarrow \infty} C^{*}(u, v) \geq 0,
$$

which proves that the function $\min \{u, v\}$ is $\mathrm{TP}_{r}$. Finally, since $r$ is arbitrary then it follows that the function $\min \{u, v\}$ is $\mathrm{TP}_{\infty}$.

By mimicking the proof of Theorem 6, we actually have the following stronger result. The proof is omitted.

Theorem 7 The copula density $c^{*}$ in (3.1) is $T P_{\infty}$.

Theorem 6 is in fact a consequence of Theorem 7 by using Lemma 3 below, but we provide a direct proof there.

Let $(X, Y) \sim H$ with marginal distributions $F$ and $G$, and copula function $C$. Using the language of reliability theory, define the survival functions

$$
\bar{F}(x)=1-F(x), \quad \bar{G}(y)=1-G(y),
$$


and

$$
\bar{H}(x, y)=\operatorname{Pr}(X>x, Y>y)=1-F(x)-G(y)+H(x, y),
$$

$x, y \in \mathbb{R}$. It follows from the definition of the copula function that $H(x, y)=$ $C(F(x), G(y))$ and $\bar{H}(x, y)=\bar{C}(\bar{F}(x), \bar{G}(y))$ for all $x, y \in \mathbb{R}$. Recently, Lin et al. [15] proved the following result.

Lemma 3 If the bivariate distribution $H$ has a $T P_{r}$ density with $r \geq 2$, then both $H$ and $\bar{H}$ are $T P_{r}$. Consequently, if $H$ has a $T P_{\infty}$ density, both $H$ and $\bar{H}$ are $T P_{\infty}$.

An immediate consequence of the last two theorems is the following result. In part (ii) of this result, we apply the fact that both $F$ and $G$ are nondecreasing, while both $\bar{F}, \bar{G}$ are non-increasing (see Marshall, et al. [19, p. $758])$.

Corollary 2 Let $C^{*}$ be the copula defined in (2.5).

(i) The survival function $\overline{C^{*}}$ is $T P_{\infty}$.

(ii) If $(X, Y) \sim H$ with copula $C^{*}$, then both $H$ and $\bar{H}$ are $T P_{\infty}$.

We next discuss some implications of the total positivity. By the results of Gross and Richards [9, Section 3, Example 3.7] we have the following inequalities.

Corollary 3 Let $(X, Y) \sim H$ with marginals $F, G$ and copula $C^{*}$ in (2.5) and $r \geq 2$. Then the matrix

$$
\left(E\left[X^{i-1} Y^{j-1}\right]\right)_{1 \leq i, j \leq r}=\left(\begin{array}{cccc}
1 & E[Y] & \cdots & E\left[Y^{r-1}\right] \\
E[X] & E[X Y] & \cdots & E\left[X Y^{r-1}\right] \\
\vdots & \vdots & & \vdots \\
E\left[X^{r-1}\right] & E\left[X^{r-1} Y\right] & \cdots & E\left[X^{r-1} Y^{r-1}\right]
\end{array}\right)
$$

is $T P_{r}$, provided the expectations exist.

Let $x_{1}<\cdots<x_{r}$ and $y_{1}<\cdots<y_{r}$. The matrix

$$
\left(\bar{H}\left(x_{i}, y_{j}\right)\right)_{1 \leq i, j \leq r}=\left(\begin{array}{ccc}
\bar{H}\left(x_{1}, y_{1}\right) & \cdots & \bar{H}\left(x_{1}, y_{r}\right) \\
\vdots & & \vdots \\
\bar{H}\left(x_{r}, y_{1}\right) & \cdots & \bar{H}\left(x_{r}, y_{r}\right)
\end{array}\right)
$$

is $T P_{r}$.

In particular, when $r=3$, it follows from (4.5) that

$$
\operatorname{det}\left(\begin{array}{ccc}
1 & E[Y] & E\left[Y^{2}\right] \\
E[X] & E[X Y] & E\left[X Y^{2}\right] \\
E\left[X^{2}\right] & E\left[X^{2} Y\right] & E\left[X^{2} Y^{2}\right]
\end{array}\right) \geq 0
$$


an inequality that is equivalent to

$$
\begin{aligned}
& E\left[X^{2} Y^{2}\right] E[X Y]-E\left[X^{2} Y\right] E\left[X Y^{2}\right]-E[X] E\left[X^{2} Y^{2}\right] E[Y] \\
& \quad+E\left[X^{2}\right] E\left[X Y^{2}\right] E[Y]+E[X] E\left[X^{2} Y\right] E\left[Y^{2}\right]-E\left[X^{2}\right] E[X Y] E\left[Y^{2}\right] \geq 0 .
\end{aligned}
$$

Let $x_{1}=-\infty<x_{2}=x<x_{3}=x^{\prime}$ and $y_{1}=-\infty<y_{2}=y<y_{3}=y^{\prime}$. Note that $\bar{F}(x)=\bar{H}(x,-\infty)$ and $\bar{G}(y)=\bar{H}(-\infty, y)$. By (4.6), the matrix

$$
\left(\begin{array}{ccc}
1 & \bar{G}(y) & \bar{G}\left(y^{\prime}\right) \\
\bar{F}(x) & \bar{H}(x, y) & \bar{H}\left(x, y^{\prime}\right) \\
\bar{F}\left(x^{\prime}\right) & \bar{H}\left(x^{\prime}, y\right) & \bar{H}\left(x^{\prime}, y^{\prime}\right)
\end{array}\right)
$$

is totally positive of order 3 . By calculating the $2 \times 2$ principal minor of this matrix, we find that $\bar{H}(x, y) \geq \bar{F}(x) \bar{G}(y)$; equivalently, $H(x, y) \geq F(x) G(y)$, $x, y \in \mathbb{R}$, i.e., the distribution function $H$ is positively quadrant dependent. Further, by calculating the determinant of this matrix, we obtain

$$
\begin{aligned}
& \bar{H}\left(x^{\prime}, y^{\prime}\right) \bar{H}(x, y)-\bar{H}\left(x^{\prime}, y\right) \bar{H}\left(x, y^{\prime}\right)-\bar{F}(x) \bar{H}\left(x^{\prime}, y^{\prime}\right) \bar{G}(y) \\
& \quad+\bar{F}\left(x^{\prime}\right) \bar{H}\left(x, y^{\prime}\right) \bar{G}(y)+\bar{F}(x) \bar{H}\left(x^{\prime}, y\right) \bar{G}\left(y^{\prime}\right)-\bar{F}\left(x^{\prime}\right) \bar{H}(x, y) \bar{G}\left(y^{\prime}\right) \geq 0
\end{aligned}
$$

for $x<x^{\prime}, y<y^{\prime}$.

We remark that more general inequalities can be deduced from [9, Example 3.11 .

\section{Moments of the B-spline functions with initial boundary}

In this section, we provide the moment formula for the B-spline functions with initial boundary at $t=0$ defined on $\mathbb{R}_{+}=[0, \infty)$. The expressions for $q_{k}$ and $r_{k}$ in Lemma 2 are obtained, in Corollary 4 below, as a consequence of the moment formula.

Let $N_{i}^{d}$ be a B-spline function of degree $d \geq 0$ on $\mathbb{R}_{+}$with knots:

$$
\underbrace{t_{-d}=\cdots=t_{-1}}_{d}=t_{0}=0<t_{1}=1<t_{2}=2<\cdots
$$

(compare with the previously studied B-spline function defined in (2.7)). Here, we have $t_{i}=(i)_{+}=\max \{i, 0\}$ and, as before, $N_{i}^{d}(t)$ is generated by the following recursion formula:

$$
N_{i}^{d}(t)=\frac{t-(i)_{+}}{(i+d)_{+}-(i)_{+}} N_{i}^{d-1}(t)+\frac{(i+d+1)_{+}-t}{(i+d+1)_{+}-(i+1)_{+}} N_{i+1}^{d-1}(t),
$$

$d \geq 1$, with initial conditions

$$
N_{i}^{0}(t)= \begin{cases}1, & i \geq 0 \text { and } t \in[i, i+1), \\ 0, & \text { otherwise. }\end{cases}
$$


For each $i \geq-d, N_{i}^{d}$ is a non-zero function with support $[\max \{i, 0\}, i+d+1]$. The recurrence (5.2) can be written more concretely as

$$
N_{i}^{d}(t)= \begin{cases}\frac{t-i}{d} N_{i}^{d-1}(t)+\frac{i+d+1-t}{d} N_{i+1}^{d-1}(t), & i \geq 0, \\ \frac{t}{i+d} N_{i}^{d-1}(t)+\frac{i+d+1-t}{i+d+1} N_{i+1}^{d-1}(t), & -d<i \leq-1, \\ (1-t) N_{-d+1}^{d-1}(t), & i=-d, \\ 0, & i<-d .\end{cases}
$$

For $h \geq 0$, denote the $h$-moment of $N_{i}^{d}$,

$$
\gamma_{i}^{d}(h):=\int_{-\infty}^{\infty} t^{h} N_{i}^{d}(t) \mathrm{d} t=\int_{\max \{i, 0\}}^{i+d+1} t^{h} N_{i}^{d}(t) \mathrm{d} t
$$

this quantity was used in the proof of Lemma 2 above. Then, we have the following recurrence relation for these moments.

$$
\gamma_{i}^{d}(h)= \begin{cases}\frac{\gamma_{i}^{d-1}(h+1)-i \gamma_{i}^{d-1}(h)}{d} & \\ \frac{(i+d+1) \gamma_{i+1}^{d-1}(h)-\gamma_{i+1}^{d-1}(h+1)}{d}, & i \geq 0, \\ \frac{\gamma_{i}^{d-1}(h+1)}{i+d} & \\ +\frac{(i+d+1) \gamma_{i+1}^{d-1}(h)-\gamma_{i+1}^{d-1}(h+1)}{i+d+1}, & -d<i<0, \\ \gamma_{-d+1}^{d-1}(h)-\gamma_{-d+1}^{d-1}(h+1), & i=-d, \\ 0, & i<-d,\end{cases}
$$

with boundary condition

$$
\gamma_{i}^{0}(h)= \begin{cases}\frac{(i+1)^{h+1}-i^{h+1}}{h+1}, & i \geq 0 \\ 0, & i<0\end{cases}
$$

The next result, which is interesting in its own right, presents the solution of the recurrence system in terms of the Stirling numbers of the second kind:

$$
S(n, k)=\frac{1}{k !} \sum_{j=0}^{k}(-1)^{j}\left(\begin{array}{l}
k \\
j
\end{array}\right)(k-j)^{n} .
$$

Here, $S(n, 0)=\delta_{n 0}, S(n, k)=0$ for $n<k$, and $0^{0} \equiv 1$ whenever it arises. Note also that $S(n, 1)=S(n, n)=1$ and $S(n, n-1)=n(n-1) / 2$. The Stirling numbers of the second kind satisfy the recurrence formula

$$
S(n+1, k)=k S(n, k)+S(n, k-1),
$$


and the identity

$$
S(n+1, k+1)=\sum_{j=k}^{n}\left(\begin{array}{c}
n \\
j
\end{array}\right) S(j, k)=\sum_{j=0}^{n-k}\left(\begin{array}{c}
n \\
n-j
\end{array}\right) S(n-j, k),
$$

which will be used later. For the identity (5.6), see Wagner [25] and the end of Remark 2 below.

Theorem 8 For $d \geq 0$, the h-moment of the B-spline function $N_{i}^{d}$ in (5.1) is of the form

$$
\gamma_{i}^{d}(h)= \begin{cases}\sum_{\ell=0}^{h} i^{\ell}\left(\begin{array}{c}
h \\
\ell
\end{array}\right) \frac{S(h+d+1-\ell, d+1)}{\left(\begin{array}{c}
h+d+1-\ell \\
d+1
\end{array}\right)}, & i \geq 0, \\
\frac{i+d+1}{d+1} \frac{S(h+i+d+1, i+d+1)}{\left(\begin{array}{c}
h+d+1 \\
d+1
\end{array}\right)}, & -d \leq i \leq 0, \\
0, & i<-d .\end{cases}
$$

Corollary 4 For $h=0,1$, we have

$$
\gamma_{i}^{d}(0)= \begin{cases}1, & i \geq 0, \\ \frac{i+d+1}{d+1}, & -d \leq i \leq 0, \\ 0, & i<-d\end{cases}
$$

and

$$
\gamma_{i}^{d}(1)= \begin{cases}\frac{d+2 i+1}{2}, & i \geq 0, \\ \frac{(i+d+1)^{2}(i+d+2)}{2(d+1)(d+2)}, & -d \leq i \leq 0, \\ 0, & i<-d .\end{cases}
$$

The formulas (5.8) and (5.9) can be applied to obtain the formula for the maximum correlation (3.5).

Corollary 5 For $i=0,1$,

$$
\gamma_{i}^{d}(h)=\frac{S(h+i+d+1, i+d+1)}{\left(\begin{array}{c}
h+d+1 \\
d+1
\end{array}\right)} .
$$


Proof. For the case $i=1$,

$$
\begin{aligned}
\gamma_{1}^{d}(h) & =\sum_{\ell=0}^{h}\left(\begin{array}{l}
h \\
\ell
\end{array}\right) \frac{S(h+d+1-\ell, d+1)}{\left(\begin{array}{c}
h+d+1-\ell \\
d+1
\end{array}\right)} \\
& =\frac{1}{\left(\begin{array}{c}
h+d+1 \\
d+1
\end{array}\right)} \sum_{\ell=0}^{h}\left(\begin{array}{c}
h+d+1 \\
h+d+1-\ell
\end{array}\right) S(h+d+1-\ell, d+1) \\
& =\frac{S(h+d+2, d+2)}{\left(\begin{array}{c}
h+d+1 \\
d+1
\end{array}\right)},
\end{aligned}
$$

by the identity (5.6).

Proof of Theorem 8. We prove the statement by mathematical induction on $d$. Note first that (5.7) with $d=0$ coincides with the boundary conditions (5.4) for all $i$ and $h$.

Suppose that (5.7) is true for the case $d-1$ and for all $i$ and $h$, then we wish to prove that it also holds true for the case $d$ and for all $i$ and $h$.

(i) For $i \geq 0$, by the assumption of induction,

$$
\gamma_{i}^{d-1}(h)=\sum_{\ell=0}^{h} i^{\ell}\left(\begin{array}{l}
h \\
\ell
\end{array}\right) \frac{S(h+d-\ell, d)}{\left(\begin{array}{c}
h+d-\ell \\
d
\end{array}\right)} .
$$

Then, we have

$$
\gamma_{i+1}^{d-1}(h)=\sum_{k=0}^{h}(i+1)^{k}\left(\begin{array}{l}
h \\
k
\end{array}\right) \frac{S(h+d-k, d)}{\left(\begin{array}{c}
h+d-k \\
d
\end{array}\right)}
$$

and by expanding $(i+1)^{k}$ using the binomial theorem, we obtain

$$
\gamma_{i+1}^{d-1}(h)=\sum_{k=0}^{h} \sum_{\ell=0}^{k} i^{\ell}\left(\begin{array}{l}
k \\
\ell
\end{array}\right)\left(\begin{array}{l}
h \\
k
\end{array}\right) \frac{S(h+d-k, d)}{\left(\begin{array}{c}
h+d-k \\
d
\end{array}\right)} .
$$

Interchanging the order of summation and using the identity,

$$
\left(\begin{array}{l}
k \\
\ell
\end{array}\right)\left(\begin{array}{l}
h \\
k
\end{array}\right)=\left(\begin{array}{l}
h \\
\ell
\end{array}\right)\left(\begin{array}{l}
h-\ell \\
k-\ell
\end{array}\right),
$$

we find that

$$
\gamma_{i+1}^{d-1}(h)=\sum_{\ell=0}^{h} i^{\ell}\left(\begin{array}{l}
h \\
\ell
\end{array}\right) \sum_{k=\ell}^{h}\left(\begin{array}{c}
h-\ell \\
k-\ell
\end{array}\right) \frac{S(h+d-k, d)}{\left(\begin{array}{c}
h+d-k \\
d
\end{array}\right)}
$$


Replacing $k$ by $k-l$, we have

$$
\gamma_{i+1}^{d-1}(h)=\sum_{\ell=0}^{h} i^{\ell}\left(\begin{array}{l}
h \\
\ell
\end{array}\right) \sum_{k=0}^{h-\ell}\left(\begin{array}{c}
h-\ell \\
k
\end{array}\right) \frac{S(h+d-\ell-k, d)}{\left(\begin{array}{c}
h+d-\ell-k \\
d
\end{array}\right)},
$$

and using the identity,

$$
\frac{\left(\begin{array}{c}
h-\ell \\
k
\end{array}\right)}{\left(\begin{array}{c}
h+d-\ell-k \\
d
\end{array}\right)}=\frac{\left(\begin{array}{c}
h+d-\ell \\
h+d-\ell-k
\end{array}\right)}{\left(\begin{array}{c}
h+d-\ell \\
d
\end{array}\right)}
$$

we deduce that

$$
\begin{aligned}
\gamma_{i+1}^{d-1}(h) & =\sum_{\ell=0}^{h} i^{\ell}\left(\begin{array}{l}
h \\
\ell
\end{array}\right) \frac{1}{\left(\begin{array}{c}
h+d-\ell \\
d
\end{array}\right)} \sum_{k=0}^{h-\ell}\left(\begin{array}{c}
h+d-\ell \\
h+d-\ell-k
\end{array}\right) S(h+d-\ell-k, d) \\
& =\sum_{\ell=0}^{h} i^{\ell}\left(\begin{array}{l}
h \\
\ell
\end{array}\right) \frac{S(h+d-\ell+1, d+1)}{\left(\begin{array}{c}
h+d-\ell \\
d
\end{array}\right)}
\end{aligned}
$$

where the last equality follows from the identity (5.6). Moreover, using the identity,

$$
\left(\begin{array}{c}
h+1 \\
\ell
\end{array}\right)=\left(\begin{array}{c}
h \\
\ell-1
\end{array}\right)+\left(\begin{array}{l}
h \\
\ell
\end{array}\right)
$$

we obtain

$$
\begin{gathered}
\gamma_{i}^{d-1}(h+1)-i \gamma_{i}^{d-1}(h) \\
=i^{h+1}+\sum_{\ell=0}^{h}\left[i^{\ell}\left(\begin{array}{c}
h+1 \\
\ell
\end{array}\right) \frac{S(h+d-\ell+1, d)}{\left(\begin{array}{c}
h+d-\ell+1 \\
d
\end{array}\right)}\right. \\
-i^{\ell+1}\left(\begin{array}{c}
h \\
\ell
\end{array}\right) \\
=i^{h+1}+\sum_{\ell=0}^{h}\left[i^{\ell}\left\{\left(\begin{array}{c}
h \\
\ell-1
\end{array}\right)+\left(\begin{array}{c}
h \\
\ell
\end{array}\right)\right\} \frac{S(h+d-\ell-\ell, d)}{(h+1, d)}\right. \\
\left(\begin{array}{c}
h+d-\ell+1 \\
d
\end{array}\right) \\
-i^{\ell+1}\left(\begin{array}{c}
h \\
\ell
\end{array}\right)
\end{gathered}
$$


Since $S(d, d)=1$ then

$$
\begin{aligned}
i^{h+1}+\sum_{\ell=0}^{h} i^{\ell}\left(\begin{array}{c}
h \\
\ell-1
\end{array}\right) \frac{S(h+d-\ell+1, d)}{\left(\begin{array}{c}
h+d-\ell+1 \\
d
\end{array}\right)} & =\sum_{\ell=1}^{h+1} i^{\ell}\left(\begin{array}{c}
h \\
\ell-1
\end{array}\right) \frac{S(h+d-\ell+1, d)}{\left(\begin{array}{c}
h+d-\ell+1 \\
d
\end{array}\right)} \\
& =\sum_{\ell=0}^{h} i^{\ell}\left(\begin{array}{c}
h \\
\ell
\end{array}\right) \frac{S(h+d-\ell, d)}{\left(\begin{array}{c}
h+d-\ell \\
d
\end{array}\right)}
\end{aligned}
$$

and substituting this result into (5.12), we obtain

$$
\gamma_{i}^{d-1}(h+1)-i \gamma_{i}^{d-1}(h)=\sum_{\ell=0}^{h} i^{\ell}\left(\begin{array}{l}
h \\
\ell
\end{array}\right) \frac{S(h+d-\ell+1, d)}{\left(\begin{array}{c}
h+d-\ell+1 \\
d
\end{array}\right)} .
$$

Similarly, it follows from (5.11) that

$$
\begin{aligned}
& \gamma_{i+1}^{d-1}(h+1)-i \gamma_{i+1}^{d-1}(h) \\
& =i^{h+1}+\sum_{\ell=0}^{h}\left[i^{\ell}\left(\begin{array}{c}
h+1 \\
\ell
\end{array}\right) \frac{S(h+d-\ell+2, d+1)}{\left(\begin{array}{c}
h+d-\ell+1 \\
d
\end{array}\right)}\right. \\
& \left.-i^{\ell+1}\left(\begin{array}{c}
h \\
\ell
\end{array}\right) \frac{S(h+d-\ell+1, d+1)}{\left(\begin{array}{c}
h+d-\ell \\
d
\end{array}\right)}\right] \\
& =\sum_{\ell=0}^{h} i^{\ell}\left(\begin{array}{l}
h \\
\ell
\end{array}\right) \frac{S(h+d-\ell+2, d+1)}{\left(\begin{array}{c}
h+d-\ell+1 \\
d
\end{array}\right)} .
\end{aligned}
$$

Hence, by substituting (5.13) and (5.14) into (5.3), we find that

$$
\begin{aligned}
\gamma_{i}^{d}(h) & =\frac{\gamma_{i}^{d-1}(h+1)-i \gamma_{i}^{d-1}(h)}{d}-\frac{\gamma_{i+1}^{d-1}(h+1)-i \gamma_{i+1}^{d-1}(h)}{d}+\frac{d+1}{d} \gamma_{i+1}^{d-1}(h) \\
= & \sum_{\ell=0}^{h} i^{\ell}\left(\begin{array}{l}
h \\
\ell
\end{array}\right)\left[\frac{S(h+d-\ell+1, d)}{d\left(\begin{array}{c}
h+d-\ell+1 \\
d
\end{array}\right)}-\frac{S(h+d-\ell+2, d+1)}{d\left(\begin{array}{c}
h+d-\ell+1 \\
d
\end{array}\right)}\right. \\
& \left.+\frac{(d+1) S(h+d-\ell+1, d+1)}{d\left(\begin{array}{c}
h+d-\ell \\
d
\end{array}\right)}\right] \\
= & \sum_{\ell=0}^{h} i^{\ell}\left(\begin{array}{c}
h \\
\ell
\end{array}\right) \frac{S(h+d-\ell+1, d+1)}{\left(\begin{array}{c}
h+d-\ell+1 \\
d+1
\end{array}\right)},
\end{aligned}
$$

where the last equality follows from the recurrence formula (5.5). 
(ii) When $-d<i<0$, by the assumption of induction,

$$
\gamma_{i}^{d-1}(h)=\frac{i+d}{d} \cdot \frac{S(h+i+d, i+d)}{\left(\begin{array}{c}
h+d \\
d
\end{array}\right)} .
$$

It then follows from (5.3) that

$$
\begin{aligned}
\gamma_{i}^{d}(h)= & \frac{\gamma_{i}^{d-1}(h+1)}{i+d}+\frac{(i+d+1) \gamma_{i+1}^{d-1}(h)-\gamma_{i+1}^{d-1}(h+1)}{i+d+1} \\
= & \frac{1}{i+d} \frac{i+d}{d} \frac{S(h+i+d+1, i+d)}{\left(\begin{array}{c}
h+d+1 \\
d
\end{array}\right)} \\
& +\frac{i+d+1}{d} \frac{S(h+i+d+1, i+d+1)}{\left(\begin{array}{c}
h+d \\
d
\end{array}\right)} \\
& -\frac{1}{i+d+1} \frac{i+d+1}{d} \frac{S(h+i+d+2, i+d+1)}{(h+d+1}\left(\begin{array}{c}
h \\
d
\end{array}\right) \\
= & \frac{i+d+1}{d+1} \frac{S(h+i+d+1, i+d+1)}{\left(\begin{array}{c}
h+d+1 \\
d+1
\end{array}\right)}
\end{aligned}
$$

Here we used the recurrence formula (5.5) with $n=h+i+d+1$ and $k=i+d+1$, viz.,

$S(h+i+d+2, i+d+1)=(i+d+1) S(h+i+d+1, i+d+1)+S(h+i+d+1, i+d)$.

(iii) When $i=-d$, by the inductive hypothesis,

$$
\gamma_{-d+1}^{d-1}(h)=\frac{h !(d-1) !}{(h+d) !} .
$$

Then, by (5.3), we have

$$
\gamma_{-d}^{d}(h)=\gamma_{-d+1}^{d-1}(h)-\gamma_{-d+1}^{d-1}(h+1)=\frac{h ! d !}{(h+d+1) !},
$$

which coincides with (5.7) with $i=-d$.

The proof is completed by induction on $d$.

Remark 2 Recall the generalized (higher-order) Bernoulli polynomial $B_{\ell}^{(x)}$ defined by the generating function

$$
\left(\frac{t}{e^{t}-1}\right)^{x}=\sum_{\ell=0}^{\infty} B_{\ell}^{(x)} \frac{t^{\ell}}{\ell !}
$$


$|t|<2 \pi, x \in \mathbb{R}$, where $B_{\ell}^{(x)}$ is a polynomial of degree $\ell$ in $x$ with rational coefficients. Neuman [20, Proposition 3.5] showed that $\gamma_{0}^{d}(h)=B_{h}^{(-(d+1))}$, from which (5.10) for $i=0$ in Corollary 5 also follows by the relationship between the Stirling number and the generalized Bernoulli polynomial:

$$
S(n+k, k)=\left(\begin{array}{c}
n+k \\
k
\end{array}\right) B_{n}^{(-k)} .
$$

The latter can be verified by (5.16) and the exponential generating function,

$$
\frac{1}{k !}\left(e^{t}-1\right)^{k}=\sum_{n=k}^{\infty} S(n, k) \frac{t^{n}}{n !}
$$

$t \in \mathbb{R}, k \geq 0$. See [3] and [2] for (5.17) and (5.18), respectively. The generating function (5.18) can also be established by induction on $k$ and this is equivalent to verifying the above useful identity (5.6).

Remark 3 By iteration, we have

$$
N_{-d}^{d}(t)=(1-t) N_{-d+1}^{d-1}(t)=\cdots=(1-t)^{d} N_{0}^{0}(t)=(1-t)^{d} \mathbb{1}_{[0,1)}(t),
$$

and hence its $h$-moment is equal to

$$
\gamma_{-d}^{d}(h)=\int_{0}^{1} t^{h}(1-t)^{d} \mathrm{~d} t=\frac{\Gamma(h+1) \Gamma(d+1)}{\Gamma(h+d+2)}=\frac{h ! d !}{(h+d+1) !},
$$

as shown in (5.15).

Remark 4 It can be shown that for $i \geq 0$,

$$
N_{i+1}^{d}(t)=N_{i}^{d}(t-1), \quad i+1 \leq t<i+d+2 .
$$

Therefore, the $h$-moment of $N_{i+1}^{d}$ can be calculated as

$$
\begin{aligned}
\gamma_{i+1}^{d}(h) & =\int_{i+1}^{i+d+2} t^{h} N_{i+1}^{d}(t) \mathrm{d} t=\int_{i+1}^{i+d+2} t^{h} N_{i}^{d}(t-1) \mathrm{d} t \\
& =\int_{i}^{i+d+1}(t+1)^{h} N_{i}^{d}(t) \mathrm{d} t=\sum_{j=0}^{h}\left(\begin{array}{c}
h \\
j
\end{array}\right) \gamma_{i}^{d}(j), \quad i \geq 0 .
\end{aligned}
$$

This is equivalent to the first formula of (5.7) (with $i \geq 0$ ). Indeed, for $i \geq 1$, it follows from (5.7) that

$$
\sum_{j=0}^{h}\left(\begin{array}{c}
h \\
j
\end{array}\right) \gamma_{i-1}^{d}(j)=\sum_{j=0}^{h}\left(\begin{array}{l}
h \\
j
\end{array}\right) \sum_{k=0}^{j}(i-1)^{k}\left(\begin{array}{l}
j \\
k
\end{array}\right) \frac{S(j+d+1-k, d+1)}{\left(\begin{array}{c}
j+d+1-k \\
d+1
\end{array}\right)}
$$


and if we now change variables from $0 \leq k \leq j \leq h$ to $0 \leq k \leq \ell \leq h$, where $\ell=h-j+k$, then we obtain

$$
\begin{aligned}
\sum_{j=0}^{h}\left(\begin{array}{l}
h \\
j
\end{array}\right) \gamma_{i-1}^{d}(j) & =\sum_{\ell=0}^{h} \sum_{k=0}^{\ell}(i-1)^{k}\left(\begin{array}{l}
\ell \\
k
\end{array}\right)\left(\begin{array}{l}
h \\
\ell
\end{array}\right) \frac{S(h+d+1-\ell, d+1)}{\left(\begin{array}{c}
h+d+1-\ell \\
d+1
\end{array}\right)} \\
& =\sum_{\ell=0}^{h} i^{\ell}\left(\begin{array}{l}
h \\
\ell
\end{array}\right) \frac{S(h+d+1-\ell, d+1)}{\left(\begin{array}{c}
h+d+1-\ell \\
d+1
\end{array}\right)} \\
& =\gamma_{i}^{d}(h) .
\end{aligned}
$$

Acknowledgments

This work was supported by JSPS KAKENHI Grants, Numbers 16K00060 and $16 \mathrm{H} 02792$.

\section{References}

1. Baker, R. An order-statistics-based method for constructing multivariate distributions with fixed marginals. Journal of Multivariate Analysis 99, 2312-2327 (2008)

2. Branson, D. Stirling numbers and Bell numbers: their role in combinatorics and probability. Mathematical Scientist 25, 1-31 (2000)

3. Carlitz, L. Note on Nörlund's Polynomial $B_{n}^{(z)}$. Proceedings of the American Mathematical Society 11, 452-455 (1960)

4. de Boor, C. On calculating with B-splines. Journal of Approximation Theory 6, 50-62 (1972)

5. de Boor, C. Total positivity of the spline collocation matrix. Indiana University Mathematics Journal 25, 541-551 (1976)

6. de Boor, C. A Practical Guide to Splines, Revised edn. Springer, New York (2001)

7. Dou, X., Kuriki, S., and Lin, G. D. Dependence structures and asymptotic properties of Baker's distributions with fixed marginals. Journal of Statistical Planning and Inference 143, 1343-1354 (2013)

8. Dou, X., Kuriki, S., Lin, G.D., and Richards, D. EM algorithms for estimating the Bernstein copula. Computational Statistics \& Data Analysis 93, 228-245 (2014)

9. Gross, K. I. and Richards, D. St. P. Algebraic methods toward higher order probability inequalities. In Stochastic Processes and Related Topics (B. Rajput et al., eds.), pp. 189211, Birkhäuser, Boston (1998)

10. Hardy, G.H., Littlewood, J.E., and Pólya, G. Some simple inequalities satisfied by convex functions. Messenger of Mathematics 58, 145-152 (1929)

11. Huang, J.S., Dou, X., Kuriki, S., and Lin, G. D. Dependence structure of bivariate order statistics with applications to Bayramoglu's distributions. Journal of Multivariate Analysis 114, 201-208 (2013)

12. Hwang, J.S. and Lin, G. D. Characterizations of distributions by linear combinations of moments of order statistics. Bulletin of the Institute of Mathematics, Academia Sinica 12, 179-202 (1984)

13. Karlin, S. Total Positivity, Vol. 1. Stanford University Press, Palo Alto, CA (1968)

14. Karlin, S. and Studden, W. J. Tchebycheff Systems: With Applications in Analysis and Statistics. Wiley, New York (1966) 
15. Lin, G. D., Dou, X., and Kuriki, S. The bivariate lack-of-memory distributions. To appear in Sankhyā Ser. A. Available at https://doi.org/10.1007/s13171-017-0119-1 (2018)

16. Lin, G. D., Dou, X., Kuriki, S., and Huang, J. S. Recent developments on the construction of bivariate distributions with fixed marginals. Journal of Statistical Distributions and Applications 1: 14 (2014)

17. Lin, G. D. and Huang, J.S. A note on the maximum correlation for Baker's bivariate distributions with fixed marginals. Journal of Multivariate Analysis 101, 2227-2233 (2010)

18. Macdonald, I. G. Symmetric Functions and Hall Polynomials, 2nd edn. Oxford University Press, New York (2015)

19. Marshall, A. W., Olkin, I., and Arnold, B. C. Inequalities: Theory of Majorization and Its Applications, 2nd edn. Springer, New York (2011)

20. Neuman, E. Moments and Fourier transforms of B-splines. Journal of Computational and Applied Mathematics 7, 51-62 (1981)

21. Nürnberger, G. Approximation by Spline Functions. Springer, New York (1989)

22. Pinkus, A. Totally Positive Matrices. Cambridge University Press, New York (2010)

23. Schumaker, L. L. Spline Functions: Basic Theory, 3rd edn. Cambridge University Press, Cambridge (2007)

24. Shen, X., Zhu, Y., and Song, L. Linear B-spline copulas with applications to nonparametric estimation of copulas. Computational Statistics \& Data Analysis 52, 3806-3819 (2008)

25. Wagner, C. G. Generalized Stirling and Lah numbers. Discrete Mathematics 160, 199218 (1996) 